\title{
Human Centered Game Design for Bioinformatics and Cyberinfrastructure Learning
}

\author{
Daniel Perry ${ }^{1}$ Cecilia Aragon ${ }^{1}$ Stephanie Cruz ${ }^{1}$ Mette A. Peters ${ }^{2}$ Jeanne Ting Chowning ${ }^{1,3}$ \\ ${ }^{1}$ University of Washington \\ ${ }^{2}$ Sage Bionetworks \\ Seattle, WA 98195 \\ Seattle, WA 98109 \\ ${ }^{3}$ Northwest Association for Biomedical Research \\ Seattle, WA 98119
}

\begin{abstract}
Engaging students in science, technology, engineering, and math (STEM) fields is critical to ensure the success of the next generation of scientists and engineers. Given that $97 \%$ of American teens play video games, there is a tremendous opportunity to facilitate interest in STEM topics through the design of engaging learning games. While a growing number of serious games have been developed for biological science and computer science learning, few address the communication and technical challenges that arise in cyberinfrastructure intensive projects, where multiple domain scientists and computer scientists collaborate. This paper describes empirical data collected during a year-long human centered game design process, in which design ideas generated by high school students were bridged with cyberinfrastructure and bioinformatics learning concepts. Our research shows that "fun" and engaging game elements are well suited for addressing the sociotechnical aspects of cyberinfrastructure projects. In this research we provide a human centered game design methodology for science educators and science game designers, as well as design implications for integrating game-based experiences into the use of large-scale shared computing resources and services.
\end{abstract}

\section{Categories and Subject Descriptors}

H.5.3. Information interfaces and presentation (e.g., HCI): Group and organization interfaces-Computer-supported cooperative work.

\section{General Terms \\ Design.}

\section{Keywords}

Game design, human centered design, bioinformatics, cyberinfrastructure.

\section{INTRODUCTION}

Studies have shown that participation in STEM fields is declining in the United States, especially for underrepresented minorities and women $[25,26]$. Given that some $97 \%$ of American teens play video or computer games [22], gaming provides a potential way to engage a diverse number of students in STEM concepts.

Permission to make digital or hard copies of all or part of this work for personal or classroom use is granted without fee provided that copies are not made or distributed for profit or commercial advantage and that copies bear this notice and the full citation on the first page. To copy otherwise, or republish, to post on servers or to redistribute to lists, requires prior specific permission and/or a fee.

XSEDE '13, July 22 - 25 2013, San Diego, CA, USA

Copyright is held by the owner/author(s). Publication rights licensed to ACM.

ACM 978-1-4503-2170-9/13/07\$15.00.
While there has been a growing body of research on the benefits of serious games, or games with a purpose [8, 13, 27], more research is needed to better understand why students find some games engaging and others boring. Additionally, while emotional state has been shown to be an important indicator of interest and engagement, in learning environments [14] and games [21], there is still further research needed to establish the role of affect and engagement within the framework of a STEM learning game.

Learning games offer a particular challenge for designers, as the fun and playfulness of the game often gets mired in the learning concepts themselves. Game scholars such as Prensky [34] readily acknowledge this challenge, noting that most learning games are "boring" and fail to reach the same level of engagement and "experience-centered "fun"” as commercial games. High school students we have worked with in our own research have echoed this feeling. As one student bluntly stated, "educational games are mostly lame." The balance between fun and learning concepts is a tenuous one, as another student noted when evaluating an early prototype of our game, saying, "I didn't feel like this was legitimate biology" but "if there was more biology it would limit the audience." As educators and game designers, how do we strike the proper balance of learning and engaging fun?

In the inaugural XSEDE (Extreme Science and Engineering Discovery Environment) Conference in 2012, closing speaker Steven Reiner called attention to the importance of story telling when communicating data and scientific discoveries, saying that, "the more we are inundated with data, the more the story - the narrative - becomes a powerful way to impart information and meaning" [28]. Games offer a powerful potential to help players better make sense of scientific data within the context of an immersive environment and narrative.

This paper addresses the ongoing design of the game MAX5, where players use cyberinfrastructure (CI) and bioinformatics tools to solve puzzles and retrieve clues to stop a deadly influenza outbreak. CI has received much attention since the National Science Foundation's (NSF) hallmark 2003 report on how it would revolutionize science and engineering through its "layer of enabling hardware, algorithms, software, communications, institutions, and personnel" [5]. In our design of the game MAX5 we were interested in how CI tools and practices can be made engaging and affectively relevant within the framework of a collaborative video game.

\section{$1.1 \mathrm{Cl}$ as a Sociotechnical System}

Attention to how social systems, organizations, and individual activities affect technical systems falls generally under the term "sociotechnical" [9]. Sociotechnical researchers have made a strong argument that an understanding of the varied user communities is critical for building collaborative cyberinfrastructure services that are useful [39]. A growing 
community of CI scholars is working to understand how collaborations between domain scientists and computer scientists inform the success of cyberinfrastructure projects [e.g., 23, 36, 37, 39]. Ribes and Lee [37] note that cyberinfrastructure collaborations are highly embedded in an understanding of the sociotechnical. In Ribes and Bowker's [36] research on a multidisciplinary collaboration for building the GeoSciences Network, they call attention to the importance of domain collaboration, defining "two axes of collaboration" between domain scientists and computer scientists, in which each have differing goals and reward systems that must be coordinated across discipline boundaries to develop appropriate scientific infrastructures and tools.

Zimmerman [39] argues that attention should be placed on several areas of sociotechnical interplay, including: users' perceived need for CI, the role and influence of all stakeholders, technologies used in CI that are built and maintained by others (the nonprimary users), and scalable coordination and feedback. It is these sociotechnical aspects of $\mathrm{CI}$ that we relate to game design outcomes in this paper.

In the design of our game, we felt it was important to not only provide learning concepts relevant to bioinformatics and CI training, but also an immersive social and narrative environment that addressed the sociotechnical considerations students would likely encounter in an actual CI project. It was also our goal to negotiate these sociotechnical aspects of CI in a manner that was highly enjoyable to players. To understand how CI tools and processes could be made engaging and enjoyable to students, we utilized an iterative and user centric design process that we describe further in the following sections.

\subsection{Human Centered Design}

While historically many technology design practices have forged the belief that humans will adapt to technologies or interfaces, a human centered design approach models technologies around users' behaviors and needs to provide more intuitive systems and interfaces [30]. Design methods are varied, and can be quantitative or qualitative in nature, but seek to collect data around users' behaviors, needs, and practices. Methods are often iterative throughout the design life cycle. Such human centered (or "user centered" as it is sometimes called) design approaches are not new to the game development process. Pagulayan et al. [31] note the importance of user centered design practices in game development, using various techniques, including: usability testing, initial experience playtesting, surveys, and what they call "deep gameplay" (or bringing in a group of users repeatedly over the game development life cycle to playtest prototypes and provide qualitative feedback).

While, such methods are still relatively new to the design of CI technologies, there are a growing number of scholars employing human centered design approaches to successfully understand and design CI tools across a variety of domains, including astrophysics [3, 4] and gravitational physics [35].

In our research we utilized human centered design practices as we designed and developed the bioinformatics and CI game MAX5. Over the course of the year-long development cycle, seven high school students engaged in participatory design sessions with a group of graduate students, and we also conducted interviews and playtesting sessions with students to generate further design outcomes to make the game more engaging.

The primary contributions of this paper are two-fold: 1) a human centered design methodology for the creation of science-based learning games, and 2) the identification of key areas of cyberinfrastructure resources and learning concepts that map particularly well to collaborative game environments. Our work also has broader implications for the design and development of a range of educational media and learning tools within the XSEDE network.

\section{RELATED WORK}

There are an increasing number of games that integrate STEM concepts into a collaborative player experience. The game and learning environment Whyville offers a learning virtual world for tweens, with 3.4 million registered users, 60 percent female, in 2009 [26]. In the game, players must work together to fight an epidemic using Center for Disease Control tools. The game is very broadly targeted, with a generic educational focus aimed more at elementary and early middle school students, including teaching writing skills, Internet safety, and basic math.

The citizen science game, Foldit, offers a way for players to engage in real science, and has allowed over 100,000 people to participate in protein folding puzzles. Foldit players were among the top competitors in the CASP08 proteomics challenge [19].

Annetta et al. [2] evaluated a crime scene investigation video game with genetics learning concepts. They found that while there was no significant difference in learning when compared to a control group, students that played the video game were significantly more engaged in the genetics content. They caution that further design and evaluation criteria are needed for learning games.

Several studies have also shown the value of game design in introducing students to computer science concepts [29, 32, 38]. Kitchen et al. noted the value of classroom games where students take on the role of processors to illustrate parallel computing concepts [20]. Tang et al. [38] explored the use of a city infrastructure virtual reality game to stimulate metacognition in engineering students. Papastergiou compared a computer game with web-based curriculum for learning computer memory concepts, finding that the game group had increased motivation and more effective learning outcomes [32].

Scratch, an interactive programming environment created by MIT's Media Lab, allowed players to generate their own games and animations using programming constructions similar to puzzle pieces [24]. Researchers found that the environment excited students and introduced them to programming logic without the burden of having to learn the syntax of a complex programming language.

There have been few, if any, game projects that we know of directly related to CI, much less the sociotechnical aspects of CI. Many science and engineering games are domain specific, which does not address one of the primary challenges in CI projects, namely, collaboration between computational and domain scientists over a long term project [36, 39].

\section{METHODOLOGY}

Data was collected using mixed method approaches including field notes from participatory design sessions, interviews with students, playtesting sessions, and design exercises.

Participatory Design: Seven high school students from Seattle area public schools (4 female, 3 male) participated as co-designers of the game. Students generated ideas for game mechanics and narrative elements, completed drawings and sketches of levels, and designed level environments using the Unity3D game engine. Participatory design sessions lasted one and a half hours and were 
held every other week (with most students participating in ten or more sessions).

Interviews: Semi-structured interviews were conducted with fifteen Seattle area high school students (ages 14-19; 6 female, 9 male) over the course of the year. Interview questions covered topics related to video gameplay and enjoyment, design ideas specific to MAX5, and questions about collaboration with peers and classmates. All students interviewed had also engaged in a playtesting session of the game MAX5.

Playtesting: Gameplay sessions lasting approximately twenty to forty minutes were conducted with forty-eight high school students (in both a lab and classroom setting). Students in these sessions played a beta version of the game, and provided feedback regarding the aspects they found enjoyable or aspects they would change.

High school students were recruited both through snowball sampling and through an email sent to institutional partners of the Northwest Association for Biomedical Research. NWABR is a non-profit organization dedicated to strengthening public trust in research through educational biomedical focused programs and outreach (http://nwabr.org), and has created a bioinformatics curriculum specifically for high school classes. Many of the bioinformatics concepts in the game were adapted from this curriculum.

Responses from the interviews and playtesting sessions were coded using a grounded theory approach to produce thematic areas. Grounded theory is a process in which codes are applied to thematically similar topics until saturation is reached and a theory regarding observed behaviors or actions is achieved [10].

\section{ABOUT THE GAME}

Players within the computer game MAX5 are offered two types of gameplay, as either 1) a biologist field agent collecting and sequencing DNA samples using bioinformatics software tools, or 2) a computer scientist making key decisions about shared resources and data allocation on a data game board (Figure 1). The game transports players into the fictional world of the Advanced Future Research Lab, a global scientific organization that is working to solve the mysterious disappearance of one of their top scientists, while preventing an influenza pandemic. Each player role receives partial information, encouraging players to collaborate and share data with each other to solve puzzles in the game.

Players engage in a series of mini-games focused on learning concepts and tools specific to their role (either bioinformatics or computer science related). Bioinformatics concepts include: the sequencing of DNA samples and queries performed using an ingame tool that simulates the National Center for Biotechnology Information's Basic Local Alignment Search Tool (BLAST) [7]. Players also use a visualization tool similar to the bioinformatics software tool Jalview [17], as a way to visualize and edit multiple sequence alignments.

The computer science mini-games are focused on learning concepts regarding high performance computing. Players make key decisions regarding computing processes and resource sharing strategies including: locking mechanisms, map reductions, efficient use of cycles, and multi-threaded access to shared resources.

Figure 1 shows a screen capture from each type of mini-game. In the figure, the biologist field agent is shown performing a BLAST to determine whether a swine should be quarantined to prevent further lethal outbreaks. The computer scientist game shows a puzzle scenario where two threads have collided trying to access the same resource, increasing the number of cycles necessary to process data. The player must prevent threads from trying to access the same resources at the same time to avoid the threads exploding and losing data.
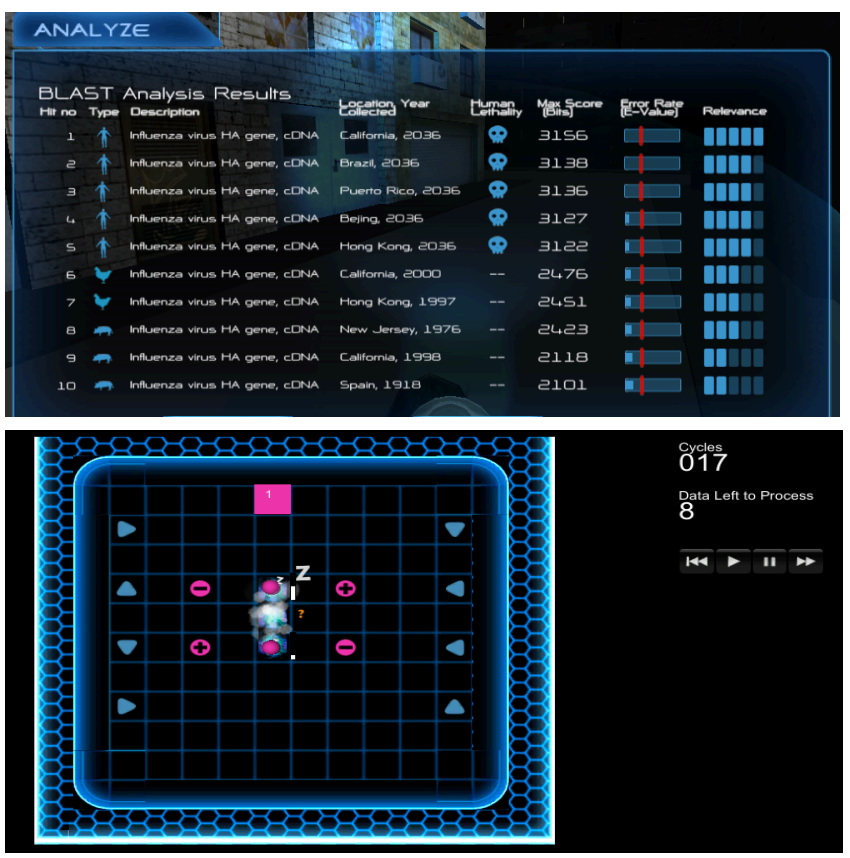

Figure 1. A player in the game MAX5 is performing a BLAST to determine an animal's flu lethality (top), and maximizing parallel processing power using a semaphore toolkit (bottom).

Players form teams consisting of one or more members of each role. Team members can communicate via a chat interface. Successful completion of each mini-game by a player increases either biological or computing resources. For example, successful completion of the computer scientist mini-games results in greater access to data, such as influenza sequence matches. All player roles are given access to a lab room where they can view outbreak patterns and team resources (Figure 2).

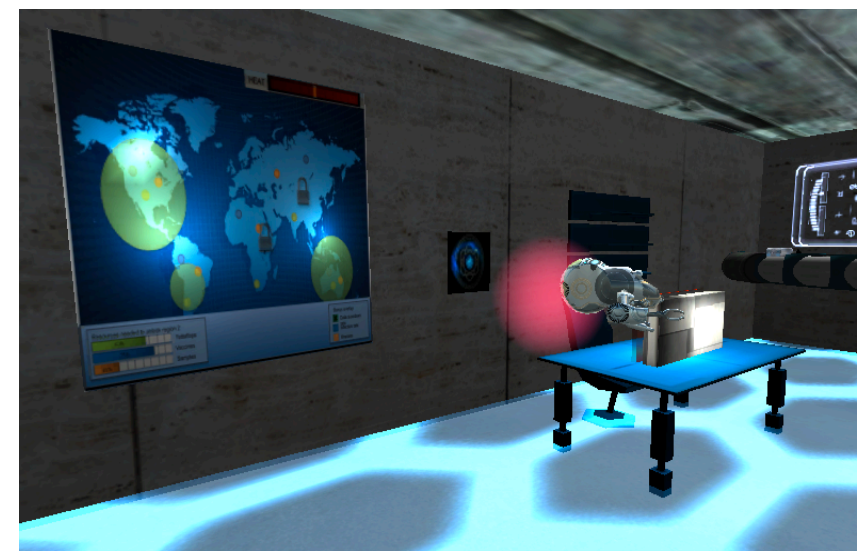

Figure 2. A design of the lab room in MAX5, used to coordinate areas of resource use. 
Design choices made regarding the type of game mechanics to include in the game, as well as ways information is shared and viewed by players, were all informed by themes that came out of our iterative design process. These themes are discussed in greater detail below.

\section{RESULTS \& DISCUSSION}

Themes that emerged from the coding of semi-structured interviews and design sessions with students clustered around several particular areas: complex coordinated communication, domain based knowledge, support for resource economies, enjoyment of tools, and diverse context-laden systems. The following sections describe our design findings and how these align with sociotechnical challenges and learning opportunities specific to CI.

\subsection{Coordinated Communication}

Designing communication tools in the game provided an opportunity to organize distributed players around team tasks, while acknowledging the inherent complexity that exists in CI intensive scientific work groups. Text-based chat was the most frequently mentioned method of interaction recommended by the students as a way to communicate with other players in-game, yet integrating real-time interactions was a continual design hurdle.

Some high school students simply were resistant or skeptical of any real time interactions in the game. One student noted, "I never got into the multiplayer thing. I liked wandering about by myself. People are annoying." Another student had a more mixed take on social interaction, saying, "I don't usually interact socially (in games). I don't enjoy listening to what people playing have to say" but then acknowledged "but they do keep it interesting and fresh." Conversely, several players said that "communicating with others" and chat were the most enjoyable aspects of game play for them. We were faced with the challenge of allowing for individual play, but of also motivating team interactions.

Coordination of actual scientists in distributed CI environment is notably also a messy process, highly dependent on the use of appropriate tools for remote communication [3, 4]. In a collaborative cross-cultural group of astrophysicists sharing supernovae data, Poon et al. found that some scientists felt more comfortable contacting supervisors directly if problems arose, while others preferred more indirect communication means via a custom designed chat interface [33]. In designing CI communication tools, how do designers account for direct and synchronous communication preferences while also accounting for players that prefer more indirect means of sharing information?

We utilized a chat interface as the primary means of communication between teammates during gameplay. Using a map in the lab room as a central visual framework within the game provided a way to share actions from geographically dispersed players, as well as a way to communicate indirectly for players that might less frequently use real time chat. The map also provided a way for players to share information about their team's shared resources without having to explicitly communicate this information to their teammates.

Design Implication: Provide both direct synchronous and indirect asynchronous means of communicating tasks and goals within the game.

\subsection{Applying Domain Based Knowledge}

In interviews, students identified numerous game genres that they found fun and engaging. Some students commented that they preferred action adventure games, or as one student succinctly put it, "teens like action games." First person shooter style games, including Halo and Call of Duty, were mentioned by many of the students as highly played. There was still also a high amount of diversity in the types of games students played. One student noted his love for strategy games, saying one of his favorite games was Age of Empires because "it's fun to build a huge army, attack other people. Start out with nothing really, and have to build resources up and gain more resources." Another student noted that while he did not really play computer games or have a console, he did play sports games like Madden with friends. Other students noted that they were experts in multiple types of games, ranging from racing, first person shooter, to puzzle. Some students were less experienced in a particular type of game play, with one young girl noting with slight embarrassment that while she played Dungeons and Dragons as a younger child, she did not play games like Halo.

The ability to shoot in-game targets, or select the best Madden NFL quarterback may not seem to be domain knowledge in the most obvious sense, but these are very much learned skills. As Gee notes, "good games are models for the production of expertise" [15]. These high school student players were therefore experts within a number of game "domains" depending on the types of games they most often played at home or with friends. Yet how might these games skills relate to CI skills?

Ribes and Lee describe the diverse set of actors in CI as coming from multiple skill areas, including domain scientists, information scientists, program officers, technicians, and multiple other actors within a highly complex eco-system [37]. These various actors must be able to communicate and apply their skills depending on the project, in a similar way to how game players must transfer their expertise between different games and differing mechanics. Kee and Browning [18] suggest that the development of CI tools is part of a "dialectic tension" between domain scientists and the technologists that develop these tools. Our design attempted to bridge the gap between technologists and domain scientists by the design of two distinct styles of play (action/ first person shooter in the case of the bioinformatics role, and puzzle logic in the computer science role).

In the design of MAX5, providing different mechanics and genre styles within the same overall game accomplished two goals: 1) it engaged players with different game skills and mechanics that they might find enjoyable and familiar based on previous games they have played; and 2) it simulated actual areas of domain knowledge that often co-exist within a CI project, in which two or more project members with differing skill sets must collaborate (e.g., a microbiologist and a computer scientist).

Design Implication: Mix differing game mechanics and genres to allow players to gain domain-specific expertise and communicate this expertise to players outside of their domain.

\subsection{Creating Resource Economies}

An interest in creating an economy for achievement, status, and rewards was a commonly requested design feature in interviews with students. Numerous students mentioned the need to integrate points for armor, leveling up, health and for purchasing tools. Giving gifts was also a practice that several students requested. One student noted that gift giving practices should be used, since they "make you feel better, when you help someone and they return the favor." Another student recommended an economic structure that encouraged collaboration in teams, with health points split in a manner that encouraged players to work together. 
Rewards and an economy within the game provided a way to help players negotiate meaning and value for the objects and actions around them.

In a similar manner, allocating and managing shared resources are of critical importance in CI projects. Grid computing networks such as the Open Science Grid provide collaborative frameworks to share and use data across multiple sites [11], however, researchers have pointed to how individual and organizational decisions about the interoperability [11], cost [6, 12], and performance $[6,12]$ of these networks determine whether and how data is actually processed and used.

In our design of MAX5, we sought to address how in-game resources could be expressed to reflect resource limitations students might encounter as part of a CI project. We accounted for computational resources by providing processing power as a limited resource that must be shared between players to perform BLAST searches or alignments (for the biologist field agent) or parallelize jobs (for the computer scientist). As an example of this resource sharing and dependencies, the number of BLAST searches or alignments the field agent is able to perform is dependent on the amount of processing power saved by players in playing the computer science mini-game.

Design Implication: Develop an in-game economy for players to make key decisions regarding the allocation of technological resources (e.g., performance speed or load balancing) as well as supporting decisions regarding the allocation of human resources (e.g., rewarding points for collaborative work).

\subsection{Enjoyment of CI Tools}

Many students expressed an explicit interest in control over their characters' interactions within the environment through the use of highly physical tools. One student noted the need to attack enemies within the game saying she wanted to "shoot them" or "the simplest thing, the cliché is to add guns." Another female student offered her opinion that "it would be cool to have some way to grab something from a distance." The comments and design ideas from students provided insights into ways we could generate engagement and enjoyment through ownership and control of tools.

Our design strategy was to embed a sense of control and enjoyment of the physical tools, and then extend the use of these tools in a manner that integrated learning components. For example, the bioinformatics tool BLAST was converted into a highly physical tool that can then be operated and literally blasted at animals as a mechanism to collect a sample sequence, and compare this sequence to matches in a database (Figure 3 ). In the computer scientist mini-games, players manipulate semaphores in the form of physical tools that can be placed on shared resource areas to restrict access and prevent these resources from exploding.

Technology acceptance theories have long stressed the importance of enjoyment and pleasure in the adoption of information technologies [1]. We argue that these theories are highly relevant to CI tools, and that CI technologies should also be enjoyable to gain traction and use by scientists and researchers. Embedding CI learning concepts into tools that produced enjoyable results in the game allowed these learning concepts to become part of an exciting and pleasurable process. Clear sensory feedback mechanisms were necessary to make the tools affectively engaging. For example, the BLAST tool produced a high-pitched zoom as it was fired, and the animals reacted with abrupt movements when enveloped in the laser sphere when "blasted".
The enjoyment of these tools was observed in playtesting sessions as students' faces lit up and smiles formed when they realized they could fire a BLAST sphere over an animal running at them. Several students commented that "it was fun to shoot" and that "the most enjoyable part of the game was shooting". Other players found the analysis aspect highly engaging, as one student noted, "analyzing the DNA (sequences) on the animals to determine whether they were lethal" was the most enjoyable part of the game.

Design Implication: Embed CI learning concepts into highly enjoyable and sensory oriented physical tools that leverage visual cues and sound feedback.

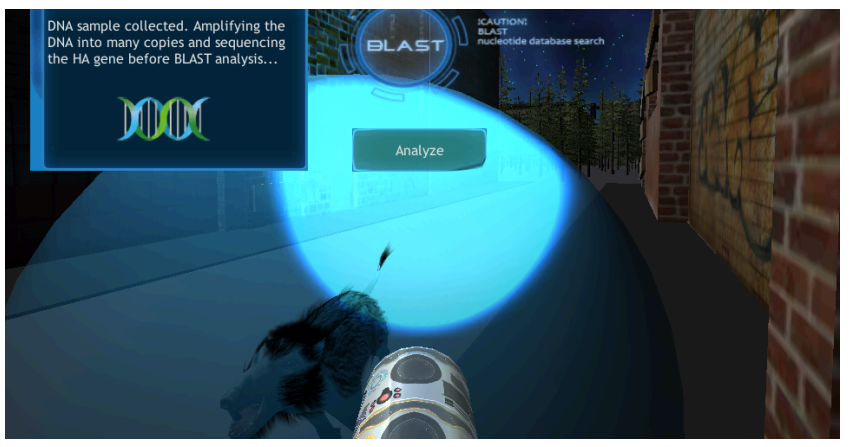

Figure 3. A screenshot of the game MAX5 as a player uses a "BLAST launcher" to collect a DNA sample from a potentially infected swine.

\subsection{Varied Needs \& Contexts}

In interviews, high school students identified a diverse number of environment contexts as important in creating engaging gameplay. One student wanted a level designed in a jungle, another an urban environment, and another flying through the air. The variations on environment were explored further with the student co-designers, each of whom designed a game level. These level designs varied greatly, with one of the student designers selecting what he describes as a "third world area" with "little actual data" and "no high-tech facilities." Another student designed her scene around a market place, in which using the BLAST tool becomes more challenging due to the player "making her way through a huge group of people who are not all infected."

Herr et al. [16] similarly discuss the importance of adaptable CI toolkits that are scalable and customizable to differing user communities in their work on the CIShell. They note that the CIShell provides tools that act as a "bridge among users, dataset and algorithm developers, and application developers" in providing a flexible and extensible framework that addresses the needs of each of these groups.

In design exercises by the high school student co-designers, they also identified diverse contexts for visualizing a BLAST output, highlighting the importance of creating flexible tools. One student focused on a quick visualization that players could use to determine a sample's lethality, using varying color and shape to mark differences. Another student designed a phylogenetic tree (Figure 4) to show sequence relationships over time, and yet another student was interested in providing a way to identify individual symptoms. Each of these methods was appropriate in addressing what students saw as their characters' needs within the game, mirroring the diverse set of needs often expressed in CI projects. 
Design Implication: Design varied level environments and multiple interaction contexts to allow players to experience CI tools as changing and adaptable.
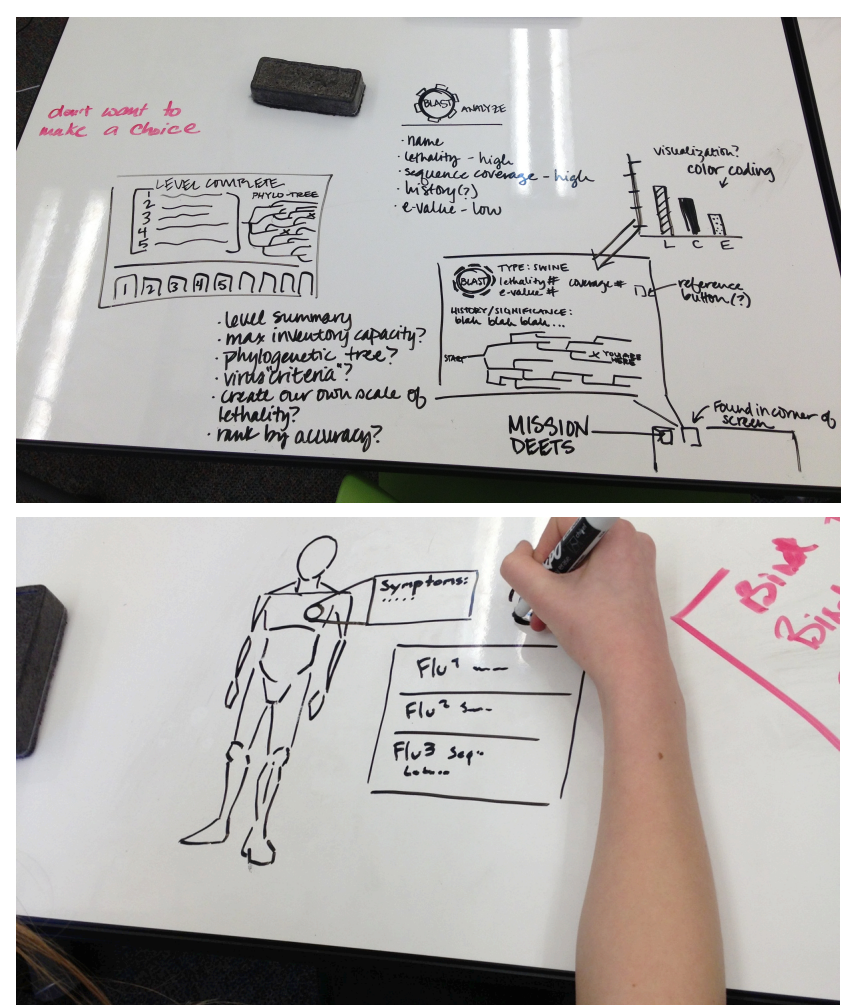

Figure 4. Two student visualizations of influenza, with one (top) focused on information organized in a broader context with samples related to each other in a phylogenetic tree, and another (bottom) focused on an individual's symptoms and personal context.

\section{FUTURE WORK}

There is still much to be learned from collecting additional data on player interactions once the game is available to a wider audience of students. In our future work, we would like to pay greater attention to the adaptability of CI environments by allowing players to customize their tools in-game for their character. This might include the use of custom BLAST interfaces, or allowing players to tweak firing range, data thread speed, or other capabilities that allow for highly adaptable play experiences.

We intend to do further studies on collaboration and engagement by analyzing the text-based interactions between players in the game. The in-game chat logs could be used to explore how players' affective phrases might reflect engagement around certain events (e.g., excitement at finding a DNA sample or solving a nucleotide puzzle clue). This information could further be used to develop AI systems that adapt to players shifting moods to encourage collaboration or to generate certain types of affect around specified events.

\section{CONCLUSION}

This research is a response to what we see as a challenge in how many science and engineering games have been designed, with a strong focus on drilling learning concepts, often at the expense of making the games exciting and emotionally engaging. If students feel that most educational games are "lame", as a high school sophomore aptly put it, this hardly seems an adequate use of a digital medium that offers unbelievably sophisticated and immersive sensory interactions and storytelling capabilities. Or as Prensky more grandly states, "video games are potentially the most engaging pastime in the history of mankind" [34]. All this leads us to wonder: how then can we as science educators and researchers rise above "lame"?

By utilizing iterative design processes that included interviews with students, playtesting sessions, and participatory design exercises, we were able to gather insights from some of the toughest (and wittiest) critics on the planet, high school students. We then worked to integrate the areas of game engagement the students identified with relevant CI practices. While we could have designed a game with the sole intent of teaching specific learning concepts, much as a textbook or educational website might (e.g., being able to search a science database, correctly comparing genetic sequences, selecting appropriate synchronization mechanisms for parallel computing), we felt this was not the most productive use of games as a medium or a fair representation of CI organizations and the real world problems that students might someday encounter.

It was our hope to generate interest and engagement in CI through our game, and indeed some of the more exciting aspects of CI are not always neat or orderly: e.g., how tools are customized and adapted, how information is communicated in a distributed group, how resources are shared between organizations and people. CI technologies do not exist in a vacuum, but are embedded in dynamic social, cultural, and political systems that are constantly evolving and changing, and we believe that games offer an ideal environment for enacting these many variable outcomes.

In this research we discussed themes that emerged from the coding of our empirical data, and identified five specific recommendations for future educational game designers who wish to integrate collaborative learning experiences into CI training. These recommendations are to: provide both direct synchronous and indirect asynchronous means of communicating tasks and goals within the game; mix differing game mechanics and genres to allow players to gain domain-specific expertise and communicate this expertise to players outside of their domain; develop an in-game economy for players to make key decisions regarding the allocation of technological resources as well as supporting decisions regarding the allocation of human resources; embed CI learning concepts into highly enjoyable and sensory oriented physical tools that leverage visual cues and sound feedback; and design varied level environments and multiple interaction contexts to allow players to experience CI tools as changing and adaptable.

These design implications are not an exhaustive list, but we hope they provide stepping stones for designers, educators, and scientists as they consider ways to make learning CI skills more playful and engaging. Science and play are after all not that dissimilar, as innovator and engineer Danny Hillis noted when discussing great scientists he has worked with, "the thing that all those people have in common is that even as adults they have an extreme sense of play" [34]. Perhaps the science and engineering community has as much to learn from youth as youth do from them about the optimal environments for collaboration and discovery. 


\section{ACKNOWLEDGMENTS}

We would like to acknowledge and thank the seven Seattle area high school students who have provided numerous insights as codesigners of the game. This work is being supported by NSF grant OCI-1135479.

\section{REFERENCES}

[1] Agarwal, R. and Karahanna, E. 2000. Time flies when you're having fun: cognitive absorption and beliefs about information technology usage. MIS quarterly, 24(4), 665694.

[2] Annetta, L.A., Minogue, J., Holmes, S.Y. and Cheng, M.T. 2009. Investigating the impact of video games on high school student's engagement and learning about genetics. Computers \& Education, 53, 74-85.

[3] Aragon, C. R., Bailey, S. J., Poon, S., Runge, K., and Thomas, R. C. 2008. Sunfall: a collaborative visual analytics system for astrophysics. Journal of Physics: Conference Series (July, 2008) Vol. 125, No. 1. IOP Publishing.

[4] Aragon, C. and Poon, S. 2011. No sense of distance: improving cross-cultural communication with context-lined software tools. In Proceedings of the 2011 iConference, 159165.

[5] Atkins, D. 2003. Revolutionizing science and engineering through cyberinfrastructure: Report of the National Science Foundation blue-ribbon advisory panel on cyberinfrastructure.

[6] Berriman, G. B., Juve, G., Deelman, E., Regelson, M., and Plavchan, P. 2010. The application of cloud computing to astronomy: A study of cost and performance. In e-Science Workshops, 2010 Sixth IEEE International Conference on (pp. 1-7). IEEE.

[7] BLAST. Retrieved March 3, 2013 from http://blast.ncbi.nlm.nih.gov/Blast.cgi

[8] Bogost. I. 2007. Persuasive Games: The expressive power of video games. MIT Press, Cambridge, MA, USA.

[9] Cherns, A. 1976. The Principles of Sociotechnical Design. Human relations, 29(8), 783-792.

[10] Corbin, J. and Strauss, A. 1990. Grounded theory research: Procedures, canons and evaluative criteria. Qualitative Sociology, 13, 3-21.

[11] de Assunção, M. D., and Buyya, R. 2009. Performance analysis of allocation policies for interGrid resource provisioning. Information and Software Technology, 51(1), $42-55$.

[12] Deelman, E., Singh, G., Livny, M., Berriman, B., and Good, J. 2008. The cost of doing science on the cloud: the montage example. In Proceedings of the 2008 ACM/IEEE conference on Supercomputing (p. 50). IEEE.

[13] Dickey, M. D. 2006. Girl gamers: the controversy of girl games and the relevance of female-oriented game design for instructional design." British journal of educational technology 37.5, 785-793.

[14] Fabri, M., Moore, D.J., Hobbs, D.J. 2004. Mediating the Expression of Emotion in Educational Collaborative Virtual Environments: An Experimental Study, in International Journal of Virtual Reality, Springer Verlag, London.
[15] Gee, J.P. 2003. What video games have to teach us about learning. New York, NY: Palgrave.

[16] Herr, B. W., Huang, W., Penumarthy, S., and Börner, K. 2006. Designing highly flexible and usable cyberinfrastructures for convergence. Annals of the New York Academy of Sciences, 1093(1), 161-179.

[17] Jalview. Retrieved April 19, 2013 from http://www.jalview.org

[18] Kee, K. F., and Browning, L. D. 2012. Two Socio-Technical Gaps of Cyberinfrastructure Development and Implementation for Data-Intensive Collaboration and Computational Simulation in Early e-Science Projects in the US. In In Proceedings of the 2012 ACM conference on Computer supported cooperative work. ACM.

[19] Khatib, F., Cooper, S., Tyka, M. D., Xu, K., Makedon, I., Popović, Z., et al. 2011. Algorithm discovery by protein folding game players. Proceedings of the National Academy of Sciences, 108(47), 18949-18953.

[20] Kitchen, A. T., Schaller, N. C., and Tymann, P. T. 1992. Game playing as a technique for teaching parallel computing concepts. ACM SIGCSE Bulletin, 24(3), 35-38.

[21] Lazzaro, N. 2008. Why we play: Affect and the fun of Games. Designing Emotions for Games, Entertainment Interfaces and Interactive Products. The Human-Computer Interaction Handbook. Fundamentals, Evolving Technologies and Emerging Applications. New York: Lawrence Erlbaum Associates.

[22] Lenhart A, Kahne J, Middaugh A, Evans C, and Vitak J. 2008. Teens, Videogames and Civics. Washington, DC: Pew Research Center. Retrieved January 10, 2013 from http://www.pewinternet.org/Reports/2008/Teens-VideoGames-and-Civics.aspx

[23] Lynch, C. 2008. The Institutional Challenges of Cyberinfrastructure and E-Research. Educause Review, 43(6), 74-76.

[24] Malan, D. J., and Leitner, H. H. 2007. Scratch for budding computer scientists. In Technical Symposium on Computer Science Education: Proceedings of the 38th SIGCSE technical symposium on Computer science education (March, 2007) Vol. 7, No. 11, pp. 223-227.

[25] Margolis, J. and Fisher, A. 2003. Unlocking the clubhouse: Women in computing. MIT Press, Cambridge, MA, USA.

[26] Mayo, M. 2007. Games for science and engineering education. In Communications of the ACM, 50(7), 31-35.

[27] McGonigal, J. 2011. Reality is Broken: Why Games Make Us Better and How They Can Change the World. Penguin, London.

[28] McKenna, S. 2012. Steven Reiner Urges Scientists to Tell Their Stories. HPCwire. Aug. 7, 2012. Retrieved March 1, 2013 from http://www.hpcwire.com/hpcwire/2012-0807/steven_reiner_urges_scientists_to_tell_their_stories.html

[29] Overmars, Mark. 2004. Teaching computer science through game design. Computer 37.4: 81-83.

[30] Oviatt, S. 2006. Human-centered design meets cognitive load theory: designing interfaces that help people think. International Multimedia Conference: Proceedings of the 14 the annual ACM international conference on Multimedia. Vol. 23. No. 27. 
[31] Pagulayan, R. J., et al. 2003. User-centered design in games. The human-computer interaction handbook: fundamentals, evolving technologies and emerging applications: 883-906.

[32] Papastergiou, M. 2009. Digital Game-Based Learning in high school Computer Science education: Impact on educational effectiveness and student motivation. Computers \& Education, 52(1), 1-12.

[33] Poon, S. S., Thomas, R. C., Aragon, C. R., and Lee, B. 2008. Context-linked virtual assistants for distributed teams: an astrophysics case study. In Proceedings of the 2008 ACM conference on Computer supported cooperative work (pp. 361-370). ACM.

[34] Prensky, M. 2001. Digital Game-Based Learning, New York, NY: McGraw-Hill.

[35] Prestopnik, N. 2013. Cooperative Visualization: A Design Case. In Proceedings of the 2013 iConference. ACM.
[36] Ribes, D. and Bowker, G. C. 2008. Organizing for Multidisciplinary Collaboration: The Case of the Geosciences Network. Scientific collaboration on the internet, 311.

[37] Ribes, D. and Lee, C. P. 2010. Sociotechnical studies of cyberinfrastructure and e-Research: Current Themes and Future Trajectories. Computer Supported Cooperative Work (CSCW), 19(3), 231-244.

[38] Tang, Y., Shetty, S., Jahan, K., Henry, J., and Hargrove, S. 2012. SustainCity-An interactive virtual reality game system. In Interdisciplinary Engineering Design Education Conference (IEDEC), 2012 2nd (pp. 18-22). IEEE.

[39] Zimmerman, A. 2007. A socio-technical framework for cyberinfrastructure design. Proceedings of e-Social Science. Ann Arbor, MI, USA, 10. 